\title{
Sex hormones, adiposity, and metabolic traits in men and women: a Mendelian randomisation study
}

\author{
Nellie Y Loh ${ }^{1}$, Edward Humphreys ${ }^{2}$, Fredrik Karpe ${ }^{1,3}$, Jeremy W Tomlinson ${ }^{1,3}$, Raymond Noordam ${ }^{4}$ and \\ Constantinos Christodoulides ${ }^{1}$
}

${ }^{1}$ Oxford Centre for Diabetes, Endocrinology and Metabolism, Radcliffe Department of Medicine, University of Oxford, Oxford, UK, ${ }^{2}$ Nuffield Department of Population Health, University of Oxford, Oxford, UK, ${ }^{3}$ NIHR Oxford Biomedical Research Centre, OUH Foundation Trust, Oxford, UK, and ${ }^{4}$ Department of Internal Medicine, Section of Gerontology and Geriatrics, Leiden University Medical Centre, Leiden, The Netherlands

Correspondence should be addressed to C Christodoulides Email

costas.christodoulides@ ocdem.ox.ac.uk

\begin{abstract}
Objective: Epidemiological and clinical studies have highlighted important roles for sex hormones in the regulation of fat distribution and systemic metabolism. We investigated the bidirectional associations between bioavailable serum testosterone (BioT) in both sexes and oestradiol (E2) in men and adiposity and metabolic traits using Mendelian randomisation (MR).

Design and Methods: As genetic instruments for sex hormones, we selected all the genome-wide significant, independent signals from a genome-wide association studies (GWAS) in up to 425097 European ancestry UK Biobank participants. European population-specific, summary-level data for adiposity, metabolic, and blood pressure traits were obtained from the largest publicly available GWAS. Sex-specific, two-sample MR analyses were used to estimate the associations of sex hormones with these traits and vice versa.

Results: In women, higher BioT was associated with obesity, upper-body fat distribution, and low HDL-cholesterol although, based on analyses modelling the sex hormone-binding globulin-independent effects of BioT, the last two associations might be indirect. Conversely, obesity and android fat distribution were associated with elevated serum BioT. In men, higher BioT was associated with lower hip circumference and lower fasting glucose. Reciprocally, obesity was associated with lower BioT and higher E2, while upper-body fat distribution and raised triglycerides were associated with lower E2.

Conclusions: Adipose tissue and metabolic dysfunction are associated with deranged sex hormone levels in both sexes. In women, elevated BioT might be a cause of obesity. Conversely, in men, higher BioT appears to have beneficial effects on adiposity and glucose metabolism.
\end{abstract}

\section{Introduction}

Body fat distribution is an independent risk factor for type 2 diabetes (T2D) and cardiovascular disease (CVD). While upper-body (android) fat accumulation, especially visceral adipose tissue (VAT), is associated with increased CVD risk due to deranged glucose and lipid metabolism, lower-body (gynoid) fat distribution provides protection against T2D and atherosclerosis (1). Compared to men, women generally have more fat mass (2). Additionally, premenopausal women typically have a greater proportion of subcutaneous AT (SAT), particularly in the gluteofemoral Printed in Great Britain

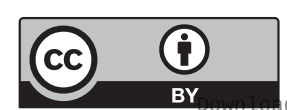

This work is licensed under a Creative Commons Attribution 4.0 International License. 
depot, while men generally accumulate more upper-body fat and have a higher proportion of VAT than women $(2,3)$. Sex differences in lipid, glycaemic, and blood pressure (BP) traits have also been described, with women having a lower CVD risk factor burden compared to men (4). Accordingly, men have an elevated risk of T2D, CVD, and all-cause mortality than women during middle age $(3,4)$.

Sex-dependent differences in fat mass and distribution are first observed during puberty (5), highlighting the importance of sex hormones in AT development and distribution. Reduced testosterone levels in men are also associated with increased upper-body fat accumulation and insulin resistance (IR) (6). However, data on testosterone replacement therapy are inconsistent in showing beneficial effects on regional adiposity (6). Similarly, a decline in oestrogen levels during menopause in women is associated with changes in fat distribution with an increase of upper-body and visceral fat mass and a reduction in gluteofemoral fat mass (7). Endogenous oestrogens (8) but not exogenous oestrogen administration (9) also appear to have beneficial effects in the regulation of adiposity, fat distribution, and CVD risk profile in men. Conversely, most observational data suggest that endogenous, physiological circulating testosterone levels are positively associated with android fat accumulation and IR in women (6). Consistent epidemiological findings have also been reported in women with hyperandrogenism secondary to polycystic ovary syndrome (PCOS) $(6,10$, $11,12,13)$. Nonetheless, there are presently no definitive data that PCOS women have increased upper-body fat and in particular, VAT compared to non-hyperandrogenic controls $(6,10,12,13,14)$. Similarly, clinical trial evidence of testosterone administration in women is insufficient to confirm the apparently harmful associations between higher testosterone and elevated cardiometabolic risk reported in observational studies $(15,16,17)$.

Mendelian randomisation (MR) is an epidemiological tool using data from genetic studies to estimate the non-confounded relationships between exposures and outcomes $(18,19)$. Several previous MR studies investigated the associations of genetically instrumented sex hormones and anthropometric, metabolic, and cardiovascular traits $(20,21,22,23)$. These studies utilised small numbers of relatively weak genetic instruments and reported negative associations between genetically instrumented sex hormone-binding globulin (SHBG) levels and T2D risk, null effects of oestradiol (E2) and testosterone on CVD risk, and an inverse association between genetically predicted higher BMI and serum testosterone in men. More recently, an MR study using many instrumental variables derived from a large GWAS in the UK Biobank (UKBB) investigated the associations of genetically influenced sex hormone levels and T2D, glycaemic, and body composition phenotypes. It was shown that higher testosterone was associated with increased lean body mass and lower T2D risk in men (24). Conversely, in women, higher testosterone increased the risk of T2D and PCOS (24). Herein, we extended these findings by conducting a bidirectional MR study to determine the associations between genetically instrumented serum testosterone in both sexes and serum E2 in men and anthropometric, glycaemic, as well as lipid and BP traits and vice versa. Where the causal relationship between two related traits is unknown, bidirectional MR can be used to determine the causal direction(s) of effect using two independent sets of genetic variants found to be associated with each trait in different GWAS. Because most circulating testosterone is bound to SHBG and is consequently biologically inactive, we focused on nonSHGB-bound (bioavailable) testosterone (BioT).

\section{Methods}

We conducted a bidirectional, two-sample MR study to investigate the relationships between circulating sex hormone levels and adiposity (BMI, waist-to-hip ratio adjusted for BMI (WHRadjBMI), BMI-adjusted waist (WCadjBMI) and hip (HIPadjBMI) circumferences), glycaemic (glucose, insulin), lipid (triglycerides, LDLcholesterol, HDL-cholesterol), and BP (systolic, diastolic) traits. As genetic instruments for sex hormones, we selected all the genome-wide significant SNPs from a GWAS conducted in up to 425097 participants of European descent from the UKBB (24). Collectively, these signals accounted for 14 and $12 \%$ of the heritability of serum BioT in women and men, respectively, and $2 \%$ of the heritability of serum E2 in men. While a GWAS of E2 levels has also been conducted in women (25), the UKBB mainly comprises of postmenopausal subjects (>75\%). Additionally, the aforementioned study (25) only identified two independent signals in women both of which became non-significant after adjustment for SHBG and testosterone levels. Consequently, we opted not to pursue women-specific E2 analyses. Since the genetic architectures of circulating BioT were separate between sexes, analyses were conducted on sex-specific outcomes. Furthermore, given the sex-specific genetic correlations between BioT and SHBG ( $\mathrm{rg}=-0.74$ in women; $\mathrm{rg}=-0.05$ in men) and between E2 and SHBG in men $(\mathrm{rg}=0.19)(24)$, we undertook additional (cluster-filtered) analyses with 
genetic instruments modelling the effects of sex hormones independent of SHBG as previously identified via cluster analyses (24). Finally, we conducted unfiltered and clusterfiltered MR analyses after excluding variants with larger effects on outcome than the tested sex hormone trait (Steiger-filtering). For outcome data, European populationspecific summary-level statistics for adiposity and metabolic traits were obtained from the largest publicly available GWAS (Supplementary Table 1, see section on supplementary materials given at the end of this article). For all the exposure instruments for which there was no direct match in the outcome datasets, we used proxy SNPs based on a LD cut-off of $\mathrm{r} 2>0.5$.

Because, no sex-stratified lipid and blood pressure GWAS have been reported to date, we also conducted new sex-specific GWAS in European ancestry participants from the UKBB using standard methodology (26) as detailed in the Supplementary Information, followed by one-sample MR. In this regard, two-sample MR methods can be reliably used for one-sample MR performed within large biobanks since they yield similar performance, in terms of bias and precision of the MR estimate, except for the MR-Egger sensitivity analysis (27).

To examine reverse causality, i.e. the potential causal effects of adiposity and metabolic traits on circulating sex hormone levels, we used genome-wide significant genetic instruments from the respective studies (Extended Tables 1). Additionally, we conducted one-sample MR studies using sex-specific genetic instruments for these traits derived from the GWAS we conducted in the UKBB (Extended Table 1). For outcome data, we utilised summary statistics from Ruth et al (24).

We employed the inverse-variance weighted (IVW) approach for two-sample MR analyses, with additional sensitivity analyses using MR-Egger, weighted-median, and MR-PRESSO (detailed in Supplementary Information). Analyses were conducted using the TwoSampleMR package (v0.5.6) implemented in R (v4.1.0) statistical software (28). Results were corrected for multiple testing with $P \leq 0.004$ $(0.05 / 12)$ considered significant. This cut-off is based on assessments of three hormone traits and four classes of cardiovascular risk factors (anthropometric, glycaemic, lipid, and blood pressure). Given the high correlation between some traits, this level of correction provides a balance between rigorous results and avoiding false negatives. A statistically significant IVW result coupled with directionally consistent associations from all three sensitivity analyses was considered as sufficient evidence to claim a causal effect.

\section{Results}

\section{MR analyses in women}

Using IVW MR, elevated serum BioT was associated with higher BMI $(\beta=0.086 \quad$ S.D., $\quad$ S.E. $=0.021$, $\left.P=3.6 \times 10^{-5}\right)$, higher WHRadjBMI $(\beta=0.115$ s.D., S.E. $\left.=0.024, \quad P=1.5 \times 10^{-6}\right)$, lower HDL-cholesterol $\left(\beta=-0.176\right.$ S.D., S.E. $\left.=0.039, P=4.8 \times 10^{-6}\right)$, and higher systolic BP ( $\beta=1.254 \mathrm{mmHg}$, S.E. $=0.400, P=0.002)$ (Fig. $1 \mathrm{~A}$ and Supplementary Table 2). Sensitivity analyses using weighted-median MR and MR-Egger (Fig. 1A and Supplementary Table 2) demonstrated uniform directions to IVW MR for all significant associations, except for that between BioT and BMI, which was directionally inconsistent in the MR-Egger model and was also associated with directional pleiotropy $($ MR-Egger intercept $=0.001$ ) Comparable results were also obtained after Steigerfiltering to exclude variants with larger effects on outcome traits than BioT apart from the association between BioT and systolic BP, which became non-significant (Supplementary Table 2). In contrast, only a borderline significant association between elevated BioT and higher BMI persisted in MR analyses using the cluster-filtered genetic instrument, modelling the SHBG-independent effects of testosterone.

MR analyses in the reverse direction revealed that genetically determined higher BMI $(\beta=0.219$ units, S.E. $=0.014, P=4.7 \times 10^{-53}$ ) and higher WHRadjBMI $\left(\beta=0.103\right.$ units, S.E. $\left.=0.016, \quad P=4.3 \times 10^{-10}\right)$ were associated with elevated serum BioT in women (Fig. 1B and Supplementary Table 3). Higher systolic BP was also associated with raised serum BioT, but the effect size was minute $(\beta=0.004$ units, S.E. $=0.001, P=0.004)$. Sensitivity analyses yielded similar results to IVW MR (Fig. 1B and Supplementary Table 3). However, they also provided evidence of unbalanced pleiotropy for the two main associations ( $p$ for pleiotropy from MR-Egger $\leq 0.01$ ). Exclusion of pleiotropic variants, however, did not materially alter the findings using IVW regression (Supplementary Table 3). Similarly, MR analyses using the Steiger-filtered genetic instruments yielded consistent findings (Supplementary Table 3).

Since the lipid and BP GWAS summary statistics were derived from sex-combined populations, we also conducted, one-sample, bidirectional MR studies using women-specific data for these traits generated from the UKBB. These analyses yielded consistent results to the two-sample MR with sex-combined data (Supplementary Table 4). The effect size of genetically instrumented BioT 
A

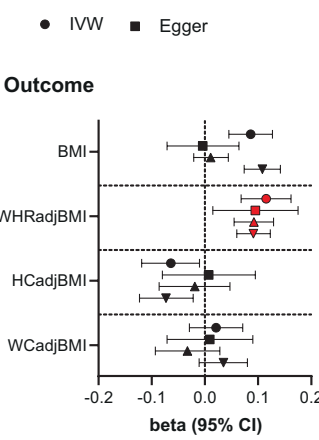

Outcome

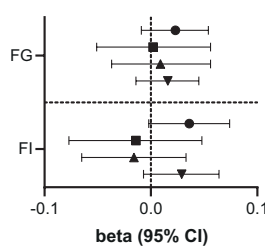

Outcome

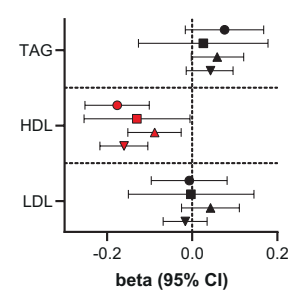

Outcome

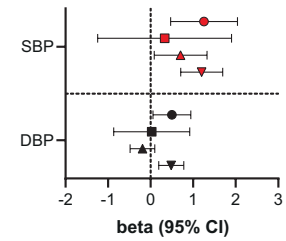

B BioT (Outcome)

A Weighted median $\checkmark$ PRESSO

Exposure

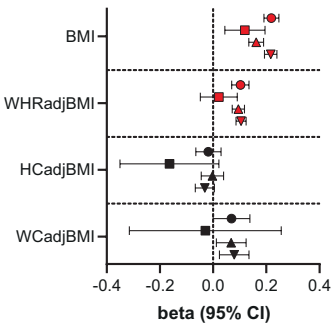

Exposure

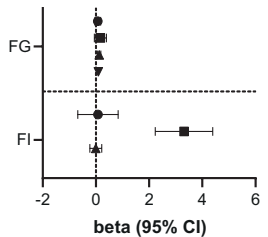

Exposure

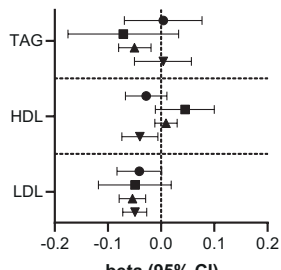

beta $(95 \% \mathrm{Cl})$

Exposure

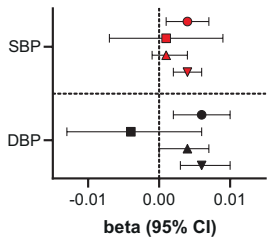

Figure 1

Bidirectional two-sample MR estimates of the relationship between bioavailable testosterone (BioT) levels and anthropometric, metabolic, and blood pressure traits in women. IVW, MR-Egger, weighted-median, and MR-PRESSO estimates (with $95 \% \mathrm{Cl}$ ) are shown for primary analyses of $(\mathrm{A})$ BioT on anthropometric, metabolic, and blood pressure traits (from Supplementary Table 2) and (B) anthropometric, metabolic, and blood pressure traits on BioT (from Supplementary Table 3). Red-filled symbols: analyses yielding IVW results with $P \leq 0.004$ (Bonferroni correction for multiple testing) and that are directionally consistent across all sensitivity analyses. WHRadjBMI, BMI-adjusted waist-to-hip ratio; HIPadjBMI, BMI-adjusted hip circumference; WCadjBMI, BMI-adjusted waist circumference; FG, fasting glucose; Fl, fasting insulin; TAG, triglycerides; HDL, HDL-cholesterol; LDL, LDL-cholesterol; SBP, systolic blood pressure; DBP, diastolic blood pressure. on systolic BP, however, was an order of magnitude lower than in the primary analysis although, the association was strengthened $\left(\beta=0.120 \mathrm{mmHg}\right.$, S.E. $\left.=0.025, P=2.1 \times 10^{-6}\right)$ and remained significant after cluster and Steiger-filtering. The association between higher genetically predicted BioT and lower HDL-cholesterol also became robust to cluster-filtering.

Finally, because the instruments for the MR analyses involving sex hormones and BMI, WHRadjBMI, and BP traits were derived from partially overlapping populations, which may lead to biased estimates, we conducted additional MR analyses studies using summary statistics for anthropometric and BP traits excluding the UKBB cohort (Supplementary Table 5). These yielded directionally consistent IVW estimates, which were at least nominally significant in the unfiltered analyses for all the main, primary associations (Supplementary Table 5).

\section{MR analyses in men}

In men, genetically predicted elevated BioT was associated with lower HIPadjBMI $(\beta=-0.127$ S.D., S.E. $=0.040$, $P=0.002)$ and a trend for lower blood glucose $(\beta=-0.064$ $\mathrm{mmol} / \mathrm{L}, \mathrm{s} . \mathrm{E} .=0.023, P=0.005)($ Fig. $2 \mathrm{~A}$ and Supplementary Table 6), while higher genetically predicted serum E2 was associated with lower WHRadjBMI $(\beta=-0.710$ S.D., S.E. $=0.241, P=0.003$ ) (Fig. $3 \mathrm{~A}$ and Supplementary Table 7). Similar results were obtained in sensitivity analyses (Supplementary Tables 6 and 7). Furthermore, directionally consistent and robust associations were derived using the cluster-filtered and Steiger-filtered sex hormone exposure instruments (Supplementary Tables 6 and 7) apart from the association between E2 and WHRadjBMI, which was nominally significant in the cluster-filtered analysis and non-significant after Steiger-filtering (Supplementary Table 7).

MR analyses in the reverse direction revealed that higher BMI was associated with lower serum BioT $\left(\beta=-0.128\right.$ units, S.E. $\left.=0.017, P=9.9 \times 10^{-15}\right)($ Fig. $2 \mathrm{~B}$ and Supplementary Table 8) and higher circulating E2 $(\beta=0.012$ units, S.E. $=0.003, P=0.0003$ ) (Fig. 3B and Supplementary Table 9) respectively. Conversely, higher WHRadjBMI $(\beta=-0.032$ units, s.E. $=0.010, \quad P=0.001)$ and elevated triglycerides $(\beta=-0.024$ units, S.E. $=0.004$, $P=1.1 \times 10^{-9}$ ) were associated with lower E2 levels (Fig. 3B and Supplementary Table 9). Sensitivity analyses demonstrated consistent directions and similar effect estimates to IVW MR (Figs 2B, 3B and Supplementary Tables 8, 9). Congruent associations were also detected following Steiger-filtering (Supplementary Tables 8 and 9). 
A BioT (Exposure)

\section{B BioT (Outcome)}

- IVW - Egger

\ Weighted median $\checkmark$ PRESSO

Outcome

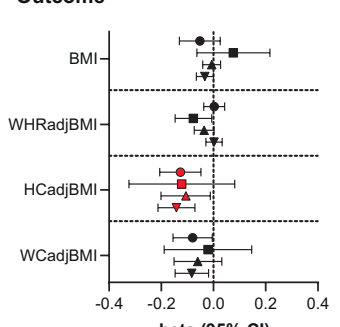

beta $(95 \% \mathrm{Cl})$

Outcome

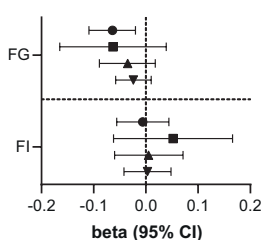

Outcome

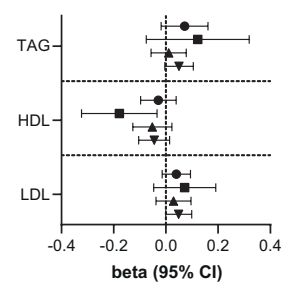

Outcome

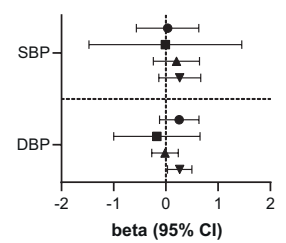

Exposure

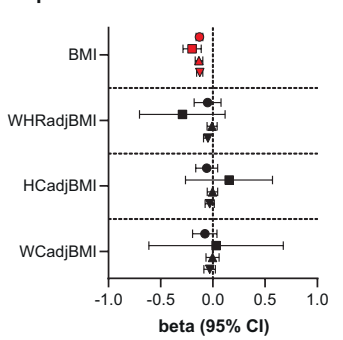

Exposure

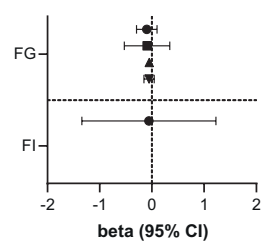

Exposure

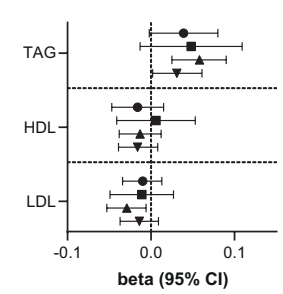

Exposure

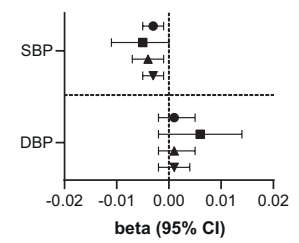

\section{Figure 2}

Bidirectional two-sample MR estimates of the relationship between bioavailable testosterone (BioT) levels and anthropometric, metabolic, and blood pressure traits in men. IVW, MR-Egger, weighted-median and MR-PRESSO estimates (with $95 \% \mathrm{Cl}$ ) are shown for primary analyses of $(\mathrm{A})$ BioT on anthropometric, metabolic, and blood pressure traits (from Supplementary Table 6) and (B) anthropometric, metabolic, and blood pressure traits on BioT (from Supplementary Table 8). Red-filled symbols: analyses yielding IVW results with $P \leq$ 0.004 (Bonferroni correction for multiple testing) and that are directionally consistent across all sensitivity analyses. WHRadjBMI, BMI-adjusted waist-to-hip ratio; HIPadjBMI, BMI-adjusted hip circumference; WCadjBMI, BMI-adjusted waist circumference; FG, fasting glucose; FI, fasting insulin; TAG, triglycerides; HDL, HDL-cholesterol; LDL, LDL-cholesterol; SBP, systolic blood pressure; DBP, diastolic blood pressure.

\section{A Oestradiol (Exposure) \\ B Oestradiol (Outcome) \\ - IVW - Egger \\ - Weighted median $\quad$ PRESSO}

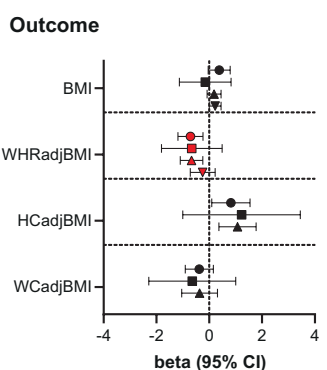

Outcome

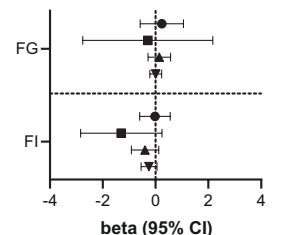

Outcome

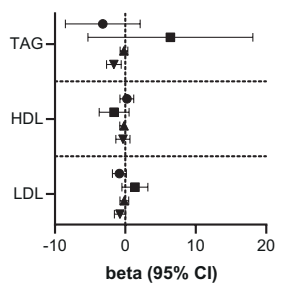

Outcome

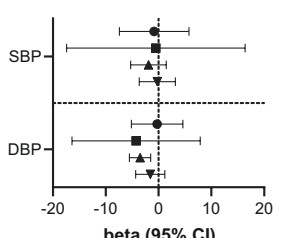

beta $(95 \% \mathrm{Cl})$
Exposure

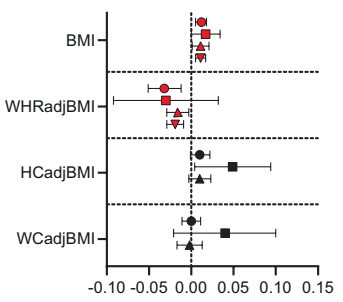

beta $(95 \% \mathrm{Cl})$

Exposure

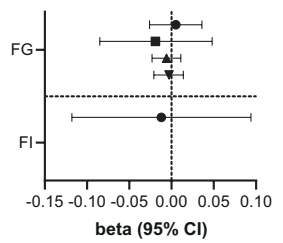

Exposure

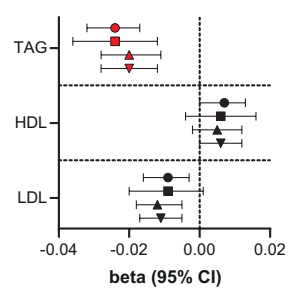

Exposure

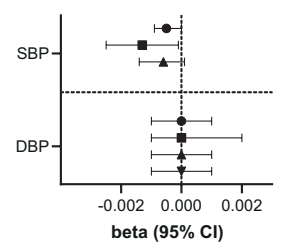

\section{Figure 3}

Bidirectional two-sample MR estimates of the relationship between oestradiol and anthropometric, metabolic, and blood pressure traits in men. IVW, MR-Egger, weighted-median, and MR-PRESSO estimates (with 95\% Cl) are shown for primary analyses of (A) oestradiol on anthropometric, metabolic, and blood pressure traits (from Supplementary Table 7) and (B) anthropometric, metabolic, and blood pressure traits on oestradiol (from Supplementary Table 9). Red-filled symbols: analyses yielding IVW results with $P \leq 0.004$ (Bonferroni correction for multiple testing) and that are directionally consistent across all sensitivity analyses. WHRadjBMI, BMIadjusted waist-to-hip ratio; HIPadjBMI, BMI-adjusted hip circumference; WCadjBMI, BMI-adjusted waist circumference; FG, fasting glucose; FI, fasting insulin; TAG, triglycerides; $\mathrm{HDL}$, HDL-cholesterol; LDL, LDL-cholesterol; SBP, systolic blood pressure; DBP, diastolic blood pressure. 
Finally, we conducted one-sample, bidirectional MR analyses between serum sex hormone levels and lipid and BP traits using male-specific data from the UKBB, which yielded directionally similar results to those obtained with two-sample MR with sex-combined data (Supplementary Table 10). Bidirectional MR analyses of sex hormones and BMI, WHRadjBMI, and BP traits using non-overlapping samples also yielded directionally consistent IVW estimates, which were at least nominally significant in the unfiltered analyses for all the main, primary associations (Supplementary Table 11).

\section{Discussion}

Our study reveals that genetically instrumented higher BioT is associated with adverse effects on fat distribution and lipid profile in women. These results extend the MR findings of Ruth et al (24), which provided suggestive evidence of a causal link between higher BioT and elevated WHRadjBMI using anthropometric data from a smaller GWAS (29). They also complement results from observational studies, which have generally reported positive associations between higher androgen levels and central obesity, CVD risk burden, and T2D risk in women $(11,12,13,30,31,32,33,34)$. The mechanisms accounting for these links are unclear. Exposure to chronic androgen excess in women with PCOS was shown to suppress SAT lipolysis in some studies $(13,35,36)$ and to impair SAT lipoprotein lipase activity, which controls the delivery of fatty acids from circulating triglyceride-rich lipoproteins to AT (13). Additionally, androgens were shown to inhibit adipogenesis of human adipose progenitors (APs) in vitro although this effect was independent of the sex or depot of origin of these cells (6). On the other hand, our cluster-filtered analyses suggest that the aforementioned associations might be indirect and driven primarily by SHBG, which may have functions beyond transporting sex steroids in blood and regulating their bioavailability and accessibility to target tissues (37). Indeed, ectopic overexpression of SHBG was shown to protect both female and male mice from genetic and diet-induced hepatosteatosis by directly reducing the production of key lipogenic enzymes in the liver (38). Additionally, SHBG treatment had anti-inflammatory and lipolytic effects on adipocytes and macrophages in vitro (39). Finally, we demonstrate a potentially direct causal link between raised serum BioT and obesity in women, which should be interpreted with caution since it was inconsistent across different MR models and was associated with directional pleiotropy.
The latter may be driven by instrumental variables being associated with inflammatory bowel disease, diuretic use, birth weight, and sleep duration. Ruth et al. also provided suggestive evidence for such an association (24). Furthermore, a meta-analysis of randomised control trials (RCTs) found that testosterone treatment resulted in postmenopausal weight gain (16).

MR analyses in the reverse direction supported an effect of obesity in driving hyperandrogenism in women. Potential sources of the horizontal pleiotropy detected for this association include effects of genetic variants on fat distribution, tendency to perform vigorous physical activity, ovarian cancer risk, and thyroid status. Similarly, observational studies have highlighted an association between raised BMI and elevated testosterone levels in women (34) and worsening of hyperandrogenism in PCOS subjects (40). This link is thought to be partly driven by the IR, which accompanies excess adiposity. Accordingly, genetic syndromes of severe IR frequently present with marked ovarian hyperandrogenism (41). Mechanistically, hyperinsulinaemia stimulates androgen production from the ovarian theca cells both directly and by increasing pituitary luteinising hormone secretion $(30,42)$. Insulin also raises BioT levels by suppressing hepatic SHBG production $(34,42,43,44)$. Unfortunately, we were unable to lend support for the role of hyperinsulinaemia in linking obesity and hyperandrogenism due to a weak insulin exposure instrument (Supplementary Table 3). AT is also capable of androgen generation and might contribute to the higher BioT levels associated with obesity. Specifically, the androgen-activating enzyme aldoketoreductase type 1C3 (AKR1C3), generates testosterone from the androgenic precursor androstenedione, is abundantly expressed in AT and its SAT expression is induced in obesity, PCOS, and hyperinsulinaemia $(35,45)$. Indeed, increased SAT AKR1C3 activity was shown to contribute to circulating androgen levels in PCOS women (35). We further show that genetically instrumented android fat distribution is also associated with raised BioT, with direct effects of genetic variants on insulin resistance, circulating leptin levels, and reproductive tract tumour susceptibility potentially accounting for the horizontal pleiotropy detected for this link. The causal association between higher WHRadjBMI and hyperandrogenism is likely partly due to the IR associated with central obesity. The conversion of androstenedione to $5 \alpha$-reduced androgens in cultures of SAT and VAT APs was also shown to be ten-fold greater than the formation of oestrone (46). Additionally, SAT $A K R 1 C 3$ expression was increased in women with android fat distribution (47). 
In contrast to women, genetically instrumented raised BioT in men was associated with decreased HIPadjBMI and lower fasting blood glucose with cluster-filtering demonstrating that these associations are likely to be driven directly by testosterone. However, no associations were detected with other metabolic or BP traits. These results are congruent with previous MR studies in the UKBB showing positive links between higher BioT and increased lean mass $(24,48)$, reduced fat mass (48), and lower glucose (24). However, they are only partly consistent with observational data demonstrating inverse associations between circulating testosterone and risk of metabolic syndrome in men $(31,32,49,50,51)$, as well as clinical findings in men with physiologic or chemically induced hypogonadism, who were shown to be at increased risk of developing obesity and IR, as well as T2DM $(8,52,53)$. On the other hand, our data are aligned with RCTs of testosterone therapy in men, which reported benefits on adiposity as reflected by lower HIPadjBMI herein, as well as diminished fasting glucose levels and T2D risk $(54,55)$. However, in contrast to our findings, a meta-analysis of these trials also showed a reduction in IR and in hypogonadal men, a decrease in triglycerides and LDL cholesterol (54). These discrepancies may be due to threshold effects of testosterone on adiposity and metabolic traits with only hypogonadal men adversely affected (6, 49). Alternatively, SHBG might also have direct effects on adiposity and metabolic traits in men. Finally, because most $(\sim 85 \%)$ circulating oestrogens in men are generated from androgens via the peripheral activity of aromatase (8), it is possible that some of the beneficial effects of testosterone may be due to conversion to E2. Consistent with this, a RCT demonstrated that the positive actions of short-term testosterone replacement on adiposity in men were driven by E2 conversion (56). Furthermore, men with congenital oestrogen deficiency due to mutations in the aromatase gene or oestrogen resistance due to oestrogen receptor loss-of-function mutations develop central obesity and hyperinsulinaemia, which, in the case of aromatase deficiency, are corrected by oestrogen replacement (57, 58, 59). Accordingly, we provide tentative evidence that genetically predicted higher E2 levels in men are associated with lower-body fat distribution. However, this finding should be cautiously interpreted as it was not robust to cluster or Steiger-filtering, which might be due to a paucity of genetic instruments in the filtered analyses. Similarly, we did not detect causal effects of E2 on metabolic traits probably because the SNPs used as instrumental variables only explained $2 \%$ of the total variation in systemic E2 levels in men (24).
While in women, obesity was associated with hyperandrogenism, genetically instrumented raised BMI in men was causally linked to low BioT and raised E2 levels. In this regard, there is a well-established epidemiological relationship between both obesity/ weight gain and hypogonadism in men, typified by low testosterone and high $\mathrm{E} 2$, which is corrected after weight loss $(34,53,60,61)$. Mechanistically, AT is enriched among metabolic tissues in CYP19A1 expression (https:// gtexportal.org), encoding aromatase and is a major source of E2 production in men. Furthermore, CYP19A1 transcript levels and activity are amplified in obesity (62, 63). Increased peripheral aromatisation of testosterone in obese men may also lead to enhanced central E2 signalling that suppresses gonadotropin production thereby exacerbating hypogonadism (8, 53, 64). Raised cortisol levels due to hypothalamo-pituitary-adrenal axis hyperactivity, systemic inflammation, hyperleptinaemia, and IR might also contribute to hypogonadism in obese men via central or testicular actions $(34,53)$. Finally, we found negative associations between genetically instrumented WHRadjBMI and hypertriglyceridaemia and E2 levels. Regarding the former, expression of CYP19A1 was shown to be lower in s.c. abdominal than gluteal AT (65). Furthermore, conversion of androgen precursors to oestrone was ten-fold higher in gluteal vs s.c. abdominal or VAT-derived APs (46). The mechanistic link between raised triglycerides and low E2 levels is unclear but might partly involve suppressed aromatase activity given that hypertriglyceridaemia was also nominally associated with raised BioT in men (Supplementary Table 7).

Our study has limitations. First, an assumption of MR is that the instrumental variables do not affect the outcome except through exposure. This issue was minimised through the use of multiple genetic variants, undertaking several sensitivity analyses and performing analyses with Steiger-filtering. Secondly, we did not employ recently developed MR methods like causal analysis using summary effect estimates (CAUSE) (66) that reduce the chances of false positives while accounting for sample overlap or Latent Heritable Confounder MR, which simultaneously estimates bidirectional causal effects and heritable confounding from GWAS summary statistics (https:// wp.unil.ch/sgg/lhc-mr/). Thirdly, the summary statistics for glycaemic traits were generated from small GWAS and explained only a small percentage of the total variance in these traits. This may account for the lack of associations between sex hormones and fasting insulin in the MR analyses and vice versa. Fourthly, our findings are based on GWAS conducted in European ancestry participants and 
hence may not extend to other ethnic populations. Finally, our data relate to lifelong exposure to sex hormones and thus may not be synonymous with the shorter-term effects of hormone replacement therapy.

In summary, we provide evidence for a bidirectional interplay between sex hormones and metabolic and adiposity traits in men and women. Our data further demonstrate that high BioT levels in women may be both a cause and consequence of obesity. In contrast, the links between hyperandrogenism and upper-body fat distribution and dyslipidaemia might be indirect and driven by lower SHBG levels. Finally, we highlight a potentially vicious cycle in men, whereby low BioT and E2 levels cause AT and metabolic dysfunction, which in turn exacerbate hypogonadism. Future experimental studies should investigate the mechanistic bases for these links.

\section{Supplementary materials}

This is linked to the online version of the paper at https://doi.org/10.1530/ EJE-21-0703.

\section{Declaration of interest}

The authors declare that there is no conflict of interest that could be perceived as prejudicing the impartiality of this study.

\section{Funding}

C C is funded by a British Heart Foundation Clinical Research Fellowship (FS/16/45/32359). F K is funded by a British Heart Foundation Programme Grant (PG/12/78/29862). J W T is supported by the Medical Research Council UK (Programme Grant MR/P011462/1).

\section{Acknowledgement}

The authors thank Jiao Luo for help with the analyses.

\section{References}

1 Manolopoulos KN, Karpe F \& Frayn KN. Gluteofemoral body fat as a determinant of metabolic health. International Journal of Obesity 2010 34 949-959. (https://doi.org/10.1038/ijo.2009.286)

2 Bredella MA. Sex differences in body composition. Advances in Experimental Medicine and Biology 2017 1043 9-27. (https://doi. org/10.1007/978-3-319-70178-3_2)

3 Huebschmann AG, Huxley RR, Kohrt WM, Zeitler P, Regensteiner JG $\&$ Reusch JEB. Sex differences in the burden of type 2 diabetes and cardiovascular risk across the life course. Diabetologia 201962 1761-1772. (https://doi.org/10.1007/s00125-019-4939-5)

4 Walli-Attaei M, Joseph P, Rosengren A, Chow CK, Rangarajan S, Lear SA, AlHabib KF, Davletov K, Dans A, Lanas F et al. Variations between women and men in risk factors, treatments, cardiovascular disease incidence, and death in 27 high-income, middle-income, and low-income countries (PURE): a prospective cohort study. Lancet 2020 396 97-109. (https://doi.org/10.1016/S0140-6736(20)30543-2)
5 Loomba-Albrecht LA \& Styne DM. Effect of puberty on body composition. Current Opinion in Endocrinology, Diabetes, and Obesity 200916 10-15. (https://doi.org/10.1097/med.0b013e328320d54c)

6 Tchernof A, Brochu D, Maltais-Payette I, Mansour MF, Marchand GB, Carreau AM \& Kapeluto J. Androgens and the regulation of adiposity and body fat distribution in humans. Comprehensive Physiology $2018 \mathbf{8}$ 1253-1290. (https://doi.org/10.1002/cphy.c170009)

7 Ambikairajah A, Walsh E, Tabatabaei-Jafari H \& Cherbuin N. Fat mass changes during menopause: a metaanalysis. American Journal of Obstetrics and Gynecology 2019221 393-409.e50. (https://doi. org/10.1016/j.ajog.2019.04.023)

8 Rubinow KB. Estrogens and body weight regulation in men. Advances in Experimental Medicine and Biology 20171043 285-313. (https://doi. org/10.1007/978-3-319-70178-3_14)

9 The Coronary Drug Project. Initial findings leading to modifications of its research protocol. JAMA 1970214 1303-1313.

10 Barber TM, Golding SJ, Alvey C, Wass JA, Karpe F, Franks S \& McCarthy MI. Global adiposity rather than abnormal regional fat distribution characterizes women with polycystic ovary syndrome. Journal of Clinical Endocrinology and Metabolism 200893 999-1004. (https://doi.org/10.1210/jc.2007-2117)

11 Moran LJ, Misso ML, Wild RA \& Norman RJ. Impaired glucose tolerance, type 2 diabetes and metabolic syndrome in polycystic ovary syndrome: a systematic review and meta-analysis. Human Reproduction Update 201016 347-363. (https://doi.org/10.1093/humupd/dmq001)

12 O'Reilly MW, Taylor AE, Crabtree NJ, Hughes BA, Capper F, Crowley RK, Stewart PM, Tomlinson JW \& Arlt W. Hyperandrogenemia predicts metabolic phenotype in polycystic ovary syndrome: the utility of serum androstenedione. Journal of Clinical Endocrinology and Metabolism 201499 1027-1036. (https://doi.org/10.1210/jc.2013-3399)

13 Manneras-Holm L, Leonhardt H, Kullberg J, Jennische E, Oden A, Holm G, Hellstrom M, Lonn L, Olivecrona G, Stener-Victorin E et al. Adipose tissue has aberrant morphology and function in PCOS: enlarged adipocytes and low serum adiponectin, but not circulating sex steroids, are strongly associated with insulin resistance. Journal of Clinical Endocrinology and Metabolism 201196 E304-E311. (https://doi. org/10.1210/jc.2010-1290)

14 O'Reilly MW, House PJ \& Tomlinson JW. Understanding androgen action in adipose tissue. Journal of Steroid Biochemistry and Molecular Biology 2014143 277-284. (https://doi.org/10.1016/j. jsbmb.2014.04.008)

15 Kalyani RR, Franco M, Dobs AS, Ouyang P, Vaidya D, Bertoni A, Gapstur SM \& Golden SH. The association of endogenous sex hormones, adiposity, and insulin resistance with incident diabetes in postmenopausal women. Journal of Clinical Endocrinology and Metabolism 200994 4127-4135. (https://doi.org/10.1210/jc.2009-0910)

16 Islam RM, Bell RJ, Green S, Page MJ \& Davis SR. Safety and efficacy of testosterone for women: a systematic review and meta-analysis of randomised controlled trial data. Lancet: Diabetes and Endocrinology 20197 754-766. (https://doi.org/10.1016/S2213-8587(19)30189-5)

17 Huang G, Tang E, Aakil A, Anderson S, Jara H, Davda M, Stroh H, Travison TG, Bhasin S \& Basaria S. Testosterone dose-response relationships with cardiovascular risk markers in androgen-deficient women: a randomized, placebo-controlled trial. Journal of Clinical Endocrinology and Metabolism 201499 E1287-E1293. (https://doi. org/10.1210/jc.2013-4160)

18 Smith GD \& Ebrahim S. 'Mendelian randomization': can genetic epidemiology contribute to understanding environmental determinants of disease? International Journal of Epidemiology 200332 1-22. (https://doi.org/10.1093/ije/dyg070)

19 Davies NM, Holmes MV \& Davey Smith G. Reading Mendelian randomisation studies: a guide, glossary, and checklist for clinicians. BMJ 2018362 k601. (https://doi.org/10.1136/bmj.k601)

20 Perry JR, Weedon MN, Langenberg C, Jackson AU, Lyssenko V, Sparso T, Thorleifsson G, Grallert H, Ferrucci L, Maggio M et al. Genetic evidence that raised sex hormone binding globulin (SHBG) 
levels reduce the risk of type 2 diabetes. Human Molecular Genetics 2010 19 535-544. (https://doi.org/10.1093/hmg/ddp522)

21 Pott J, Bae YJ, Horn K, Teren A, Kuhnapfel A, Kirsten H, Ceglarek U, Loeffler M, Thiery J, Kratzsch J et al. Genetic association study of eight steroid hormones and implications for sexual dimorphism of coronary artery disease. Journal of Clinical Endocrinology and Metabolism 2019104 5008-5023. (https://doi.org/10.1210/jc.201900757)

22 Eriksson J, Haring R, Grarup N, Vandenput L, Wallaschofski H, Lorentzen E, Hansen T, Mellstrom D, Pedersen O, Nauck M et al. Causal relationship between obesity and serum testosterone status in men: a bi-directional Mendelian randomization analysis. PLOS ONE 201712 e0176277. (https://doi.org/10.1371/journal.pone.0176277)

23 Ding EL, Song Y, Manson JE, Hunter DJ, Lee CC, Rifai N, Buring JE, Gaziano JM \& Liu S. Preparation and application of an affinity matrix for phosphatidylinositol-specific phospholipase C. New England Journal of Medicine 2009361 1152-1163. (https://doi.org/10.1016/00093084(90)90098-c)

24 Ruth KS, Day FR, Tyrrell J, Thompson DJ, Wood AR, Mahajan A, Beaumont RN, Wittemans L, Martin S, Busch AS et al. Using human genetics to understand the disease impacts of testosterone in men and women. Nature Medicine 202026 252-258. (https://doi.org/10.1038/ s41591-020-0751-5)

25 Schmitz D, Ek WE, Berggren E, Hoglund J, Karlsson T \& Johansson Å. Genome-wide association study of estradiol levels and the causal effect of estradiol on bone mineral density. Journal of Clinical Endocrinology and Metabolism 2021106 e4471-e4486. (https://doi.org/10.1210/ clinem/dgab507)

26 Loh PR, Tucker G, Bulik-Sullivan BK, Vilhjalmsson BJ, Finucane HK, Salem RM, Chasman DI, Ridker PM, Neale BM, Berger B et al. Efficient Bayesian mixed-model analysis increases association power in large cohorts. Nature Genetics 201547 284-290. (https://doi.org/10.1038/ ng.3190)

27 Minelli C, Del Greco F, van der Plaat DA, Bowden J, Sheehan NA $\&$ Thompson J. The use of two-sample methods for Mendelian randomization analyses on single large datasets. International Journal of Epidemiology 202150 1651-1659. (https://doi.org/10.1093/ije/ dyab084)

28 Hemani G, Zheng J, Elsworth B, Wade KH, Haberland V, Baird D, Laurin C, Burgess S, Bowden J, Langdon R et al. The MR-base platform supports systematic causal inference across the human phenome. eLife 20187 e34408. (https://doi.org/10.7554/eLife.34408)

29 Shungin D, Winkler TW, Croteau-Chonka DC, Ferreira T, Locke AE, Magi R, Strawbridge RJ, Pers TH, Fischer K, Justice AE et al. New genetic loci link adipose and insulin biology to body fat distribution. Nature 2015518 187-196. (https://doi.org/10.1038/nature14132)

30 Kempegowda P, Melson E, Manolopoulos KN, Arlt W \& O'Reilly MW. Implicating androgen excess in propagating metabolic disease in polycystic ovary syndrome. Therapeutic Advances in Endocrinology and Metabolism 202011 2042018820934319. (https://doi. org/10.1177/2042018820934319)

31 Lutz SZ, Wagner R, Fritsche L, Peter A, Rettig I, Willmann C, Fehlert E, Martus P, Todenhofer T, Stefan N et al. Sex-specific associations of testosterone with metabolic traits. Frontiers in Endocrinology 201910 90. (https://doi.org/10.3389/fendo.2019.00090)

32 O'Reilly MW, Glisic M, Kumarendran B, Subramanian A, Manolopoulos KN, Tahrani AA, Keerthy D, Muka T, Toulis KA, Hanif W et al. Serum testosterone, sex hormone-binding globulin and sexspecific risk of incident type 2 diabetes in a retrospective primary care cohort. Clinical Endocrinology 201990 145-154. (https://doi. org/10.1111/cen.13862)

33 Rasmussen JJ, Selmer C, Frossing S, Schou M, Faber J, Torp-Pedersen C, Gislason GH, Kober L, Hougaard DM, Cohen AS et al. Endogenous testosterone levels are associated with risk of type 2 diabetes in women without established comorbidity. Journal of the Endocrine Society 20204 bvaa050. (https://doi.org/10.1210/jendso/bvaa050)
34 Pasquali R. Obesity and androgens: facts and perspectives. Fertility and Sterility 200685 1319-1340. (https://doi.org/10.1016/j. fertnstert.2005.10.054)

35 O'Reilly MW, Kempegowda P, Walsh M, Taylor AE, Manolopoulos KN, Allwood JW, Semple RK, Hebenstreit D, Dunn WB, Tomlinson JW et al. AKR1C3-mediated adipose androgen generation drives lipotoxicity in women with polycystic ovary syndrome. Journal of Clinical Endocrinology and Metabolism 2017102 3327-3339. (https://doi. org/10.1210/jc.2017-00947)

36 Faulds G, Ryden M, Ek I, Wahrenberg H \& Arner P. Mechanisms behind lipolytic catecholamine resistance of subcutaneous fat cells in the polycystic ovarian syndrome. Journal of Clinical Endocrinology and Metabolism 200388 2269-2273. (https://doi.org/10.1210/jc.2002021573)

37 Rosner W, Hryb DJ, Kahn SM, Nakhla AM \& Romas NA. Interactions of sex hormone-binding globulin with target cells. Molecular and Cellular Endocrinology 2010316 79-85. (https://doi.org/10.1016/j. mce.2009.08.009)

38 Saez-Lopez C, Barbosa-Desongles A, Hernandez C, Dyer RA, Innis SM, Simo R \& Selva DM. Sex hormone-binding globulin reduction in metabolic disorders may play a role in NAFLD development. Endocrinology 2017158 545-559. (https://doi.org/10.1210/en.20161668)

39 Yamazaki H, Kushiyama A, Sakoda H, Fujishiro M, Yamamotoya T, Nakatsu Y, Kikuchi T, Kaneko S, Tanaka H \& Asano T. Protective effect of sex hormone-binding globulin against metabolic syndrome: in vitro evidence showing anti-inflammatory and lipolytic effects on adipocytes and macrophages. Mediators of Inflammation 20182018 3062319. (https://doi.org/10.1155/2018/3062319)

40 Lim SS, Norman RJ, Davies MJ \& Moran LJ. The effect of obesity on polycystic ovary syndrome: a systematic review and meta-analysis. Obesity Reviews 201314 95-109. (https://doi.org/10.1111/j.1467789X.2012.01053.x)

41 Semple RK, Savage DB, Cochran EK, Gorden P \& O'Rahilly S. Genetic syndromes of severe insulin resistance. Endocrine Reviews 201132 498-514. (https://doi.org/10.1210/er.2010-0020)

42 Sanchez-Garrido MA \& Tena-Sempere M. Metabolic dysfunction in polycystic ovary syndrome: pathogenic role of androgen excess and potential therapeutic strategies. Molecular Metabolism 202035100937. (https://doi.org/10.1016/j.molmet.2020.01.001)

43 Pasquali R, Casimirri F, De Iasio R, Mesini P, Boschi S, Chierici R, Flamia R, Biscotti M \& Vicennati V. Insulin regulates testosterone and sex hormone-binding globulin concentrations in adult normal weight and obese men. Journal of Clinical Endocrinology and Metabolism 199580 654-658. (https://doi.org/10.1210/ jcem.80.2.7852532)

44 Katsuki A, Sumida Y, Murashima S, Fujii M, Ito K, Tsuchihashi K, Murata K, Yano Y \& Shima T. Acute and chronic regulation of serum sex hormone-binding globulin levels by plasma insulin concentrations in male noninsulin-dependent diabetes mellitus patients. Journal of Clinical Endocrinology and Metabolism 199681 2515-2519. (https://doi.org/10.1210/jcem.81.7.8675570)

45 Quinkler M, Sinha B, Tomlinson JW, Bujalska IJ, Stewart PM \& Arlt W. Androgen generation in adipose tissue in women with simple obesity - a site-specific role for 17beta-hydroxysteroid dehydrogenase type 5. Journal of Endocrinology 2004183 331-342. (https://doi.org/10.1677/ joe.1.05762)

46 Killinger DW, Perel E, Danilescu D, Kharlip L \& Lindsay WR. Influence of adipose tissue distribution on the biological activity of androgens. Annals of the New York Academy of Sciences 1990595 199-211. (https:// doi.org/10.1111/j.1749-6632.1990.tb34293.x)

47 Ostinelli G, Vijay J, Vohl MC, Grundberg E \& Tchernof A. AKR1C2 and AKR1C3 expression in adipose tissue: association with body fat distribution and regulatory variants. Molecular and Cellular Endocrinology 2021527 111220. (https://doi.org/10.1016/j. mce.2021.111220) 
48 Mohammadi-Shemirani P, Chong M, Pigeyre M, Morton RW, Gerstein HC \& Pare G. Effects of lifelong testosterone exposure on health and disease using Mendelian randomization. eLife 20209 e58914. (https://doi.org/10.7554/eLife.58914)

49 Ottarsdottir K, Nilsson AG, Hellgren M, Lindblad U \& Daka B. The association between serum testosterone and insulin resistance: a longitudinal study. Endocrine Connections 20187 1491-1500. (https:// doi.org/10.1530/EC-18-0480)

50 Kupelian V, Page ST, Araujo AB, Travison TG, Bremner WJ \& McKinlay JB. Low sex hormone-binding globulin, total testosterone, and symptomatic androgen deficiency are associated with development of the metabolic syndrome in nonobese men. Journal of Clinical Endocrinology and Metabolism 200691 843-850. (https://doi. org/10.1210/jc.2005-1326)

51 Laaksonen DE, Niskanen L, Punnonen K, Nyyssonen K, Tuomainen TP, Valkonen VP, Salonen R \& Salonen JT. Testosterone and sex hormonebinding globulin predict the metabolic syndrome and diabetes in middle-aged men. Diabetes Care 200427 1036-1041. (https://doi. org/10.2337/diacare.27.5.1036)

52 Dhindsa S, Ghanim H, Batra M, Kuhadiya ND, Abuaysheh S, Sandhu S, Green K, Makdissi A, Hejna J, Chaudhuri A et al. Insulin resistance and inflammation in hypogonadotropic hypogonadism and their reduction after testosterone replacement in men with type 2 diabetes. Diabetes Care 201639 82-91. (https://doi.org/10.2337/dc15-1518)

53 Kelly DM \& Jones TH. Testosterone: a metabolic hormone in health and disease. Journal of Endocrinology 2013217 R25-R45. (https://doi. org/10.1530/JOE-12-0455)

54 Corona G, Giagulli VA, Maseroli E, Vignozzi L, Aversa A, Zitzmann M, Saad F, Mannucci E \& Maggi M. THERAPY OF ENDOCRINE DISEASE: Testosterone supplementation and body composition: results from a meta-analysis study. European Journal of Endocrinology 2016174 R99-R116. (https://doi.org/10.1530/EJE-15-0262)

55 Wittert G, Bracken K, Robledo KP, Grossmann M, Yeap BB, Handelsman DJ, Stuckey B, Conway A, Inder W, McLachlan R et al. Testosterone treatment to prevent or revert type 2 diabetes in men enrolled in a lifestyle programme (T4DM): a randomised, doubleblind, placebo-controlled, 2-year, phase $3 \mathrm{~b}$ trial. Lancet: Diabetes and Endocrinology $2021932-45$. (https://doi.org/10.1016/S22138587(20)30367-3)

56 Finkelstein JS, Lee H, Burnett-Bowie SA, Pallais JC, Yu EW, Borges LF, Jones BF, Barry CV, Wulczyn KE, Thomas BJ et al. Gonadal steroids and body composition, strength, and sexual function in men. New England Journal of Medicine 2013369 1011-1022. (https://doi.org/10.1056/ NEJMoa1206168)

57 Maffei L, Rochira V, Zirilli L, Antunez P, Aranda C, Fabre B, Simone ML, Pignatti E, Simpson ER, Houssami S et al. A novel compound heterozygous mutation of the aromatase gene in an adult man: reinforced evidence on the relationship between congenital oestrogen deficiency, adiposity and the metabolic syndrome. Clinical Endocrinology 200767 218-224. (https://doi.org/10.1111/j.13652265.2007.02864.x)

58 Bilezikian JP, Morishima A, Bell J \& Grumbach MM. Increased bone mass as a result of estrogen therapy in a man with aromatase deficiency. New England Journal of Medicine 1998339 599-603. (https:// doi.org/10.1056/NEJM199808273390905)

59 Smith EP, Boyd J, Frank GR, Takahashi H, Cohen RM, Specker B, Williams TC, Lubahn DB \& Korach KS. Estrogen resistance caused by a mutation in the estrogen-receptor gene in a man. New England Journal of Medicine 1994331 1056-1061. (https://doi.org/10.1056/ NEJM199410203311604)

60 Travison TG, Araujo AB, Kupelian V, O'Donnell AB \& McKinlay JB. The relative contributions of aging, health, and lifestyle factors to serum testosterone decline in men. Journal of Clinical Endocrinology and Metabolism 200792 549-555. (https://doi.org/10.1210/jc.20061859)

61 Hammoud A, Gibson M, Hunt SC, Adams TD, Carrell DT, Kolotkin RL \& Meikle AW. Effect of Roux-en-Y gastric bypass surgery on the sex steroids and quality of life in obese men. Journal of Clinical Endocrinology and Metabolism 200994 1329-1332. (https://doi. org/10.1210/jc.2008-1598)

62 Civelek M, Wu Y, Pan C, Raulerson CK, Ko A, He A, Tilford C, Saleem NK, Stancakova A, Scott LJ et al. Genetic regulation of adipose gene expression and cardio-metabolic traits. American Journal of Human Genetics 2017100 428-443. (https://doi.org/10.1016/j. ajhg.2017.01.027)

63 Cohen PG. Aromatase, adiposity, aging and disease. The hypogonadal-metabolic-atherogenic-disease and aging connection. Medical Hypotheses 200156 702-708. (https://doi.org/10.1054/ mehy.2000.1169)

64 Mah PM \& Wittert GA. Obesity and testicular function. Molecular and Cellular Endocrinology 2010316 180-186. (https://doi.org/10.1016/j. mce.2009.06.007)

65 Bulun SE \& Simpson ER. Competitive reverse transcription-polymerase chain reaction analysis indicates that levels of aromatase cytochrome P450 transcripts in adipose tissue of buttocks, thighs, and abdomen of women increase with advancing age. Journal of Clinical Endocrinology and Metabolism $1994 \mathbf{7 8}$ 428-432. (https://doi.org/10.1210/ jcem.78.2.8106632)

66 Morrison J, Knoblauch N, Marcus JH, Stephens M \& He X. Mendelian randomization accounting for correlated and uncorrelated pleiotropic effects using genome-wide summary statistics. Nature Genetics 202052 740-747. (https://doi.org/10.1038/s41588-020-0631-4)

Received 2 July 2021

Revised version received 7 January 2022

Accepted 20 January 2022 